\title{
Liquid compressibility effects during the collapse of a single cavitating bubble
}

\author{
D. Fuster ${ }^{a}$ \\ Division of Engineering and Applied Science, California Institute of Technology, Pasadena, California 91125 \\ C. Dopazo and G. Hauke \\ Laboratorio de Investigacion en Tecnologias de la Combustion-Area de Mecanica de Fluidos, Centro \\ Politecnico Superior, Universidad de Zaragoza, C/Maria de Luna 3, 50018 Zaragoza, Spain
}

(Received 23 December 2009; revised 17 September 2010; accepted 22 September 2010)

\begin{abstract}
The effect of liquid compressibility on the dynamics of a single, spherical cavitating bubble is studied. While it is known that compressibility damps the amplitude of bubble rebounds, the extent to which this effect is accurately captured by weakly compressible versions of the Rayleigh-Plesset equation is unclear. To clarify this issue, partial differential equations governing conservation of mass, momentum, and energy are numerically solved both inside the bubble and in the surrounding compressible liquid. Radiated pressure waves originating at the unsteady bubble interface are directly captured. Results obtained with Rayleigh-Plesset type equations accounting for compressibility effects, proposed by Keller and Miksis [J. Acoust. Soc. Am. 68, 628-633 (1980)], Gilmore, and Tomita and Shima [Bull. JSME 20, 1453-1460 (1977)], are compared with those resulting from the full model. For strong collapses, the solution of the latter reveals that an important part of the energy concentrated during the collapse is used to generate an outgoing pressure wave. For the examples considered in this research, peak pressures are larger than those predicted by Rayleigh-Plesset type equations, whereas the amplitudes of the rebounds are smaller. (C) 2011 Acoustical Society of America.
\end{abstract}

[DOI: 10.1121/1.3502464]

PACS number(s): 43.35.Ei, 43.25.Yw, 43.35.Hl, 43.25.Gf [DLM]

Pages: 122-131

\section{INTRODUCTION}

Different processes taking place during the collapse of cavitating bubbles have attracted the attention of the scientific and industrial communities for many applications. At implosion, pressures of the order of thousands of atmospheres and temperatures of thousands of degrees are reached inside the bubble, promoting chemical and physical processes not only inside the bubble but also in the surrounding liquid. The role of the liquid on those processes has been widely studied by several authors. Besant ${ }^{1}$ found the radial dependence in an infinite mass of an incompressible liquid of the instantaneous pressure alteration with respect to its constant value very far away from a spherical cavity as it is filled up. Rayleigh ${ }^{2}$ esti- $^{-}$ mated that before complete collapse the pressure near the boundary becomes very great. Even at the implosion of vapor and/or gas containing cavities, large pressures can also be attained under some conditions within the bubble, and also in the liquid. As a bubble collapses, its interface moves inward at high velocities and this motion produces an intense compression of the gas/vapor mixture which, simultaneously, increases its temperature, inducing dissociation and chemical reactions. For example, near the implosion of a $10 \mu \mathrm{m}$ diameter bubble, the kinetic energy of the surrounding liquid, when the bubble compresses at $100 \mathrm{~m} / \mathrm{s}$ is on the order of $10^{-8}$ and $10^{-7} \mathrm{~J}$, which must be brought to a stop through a pressure increment at the cavity interface between hundreds and thousands of atmospheres, respectively. This situation bears a

\footnotetext{
a) Author to whom correspondence should be addressed. Electronic mail: fuster@caltech.edu
}

strong resemblance to the waterhammer phenomenon in a duct: As the liquid flow is halted by the abrupt closing of a valve, pressure waves propagate upstream, reflect at the duct inlet, travel downstream to the valve, this sequence repeating hereafter. The role of the valve is played in the present case by the bubble interface, which opposes the inward liquid flow.

Experiments on single bubbles do often take place inside spherical containers. A transducer, with a well defined frequency, causes the vibration of the outer vessel wall. In the absence of a bubble, standing waves are generated. A single bubble of radius $R_{0}$, much smaller than the forcing wavelength, can be made to oscillate under the externally imposed pressure field.

The Rayleigh-Plesset (RP) equation, and its weakly compressible generalizations, such as the Gilmore ${ }^{3}$ and KellerMiksis (KM) equations, ${ }^{4}$ have been widely used to investigate single bubble dynamics. ${ }^{5-11}$ The validity of different models based on the RP equations accounting for compressibility effects has been discussed. ${ }^{12-15}$ Moreover, these studies are focused on the bubble interior behavior. Analysis of the liquid dynamics during the bubble collapse in scarce. ${ }^{16,17}$ The works of Prosperetti and Lezzi, ${ }^{18,19}$ theoretically quantifying the order of accuracy of various RP-type equations as a function of the Mach number, are relevant examples.

Johnson and Colonius ${ }^{17,20}$ have developed numerical methods in order to capture pressure waves generated by non-spherical bubble implosion in the liquid. However, a numerical study of the validity of RP-type equations to predict strong spherical bubble collapses is missing.

Pressure waves emitted in single bubble sonoluminescence (SBSL) have been experimentally measured by 
Wang et al. ${ }^{21}$ and Pecha and Gompf, ${ }^{22}$ but the lack of realistic models prevents establishing a correlation between the pressure values far away from the bubble and those encountered inside the bubble during the implosion. Moreover, in multi-bubble systems pressure waves could interact with the bubble cloud; ${ }^{23}$ although they should not significantly alter the dynamics of the individual surrounding bubbles, this interaction may, under some conditions, generate in the liquid strong shock waves propagating inward to the cloud center. $^{24,25}$

In order to quantify the accuracy of widely used RP-type equations, a new model is formulated, improving the predictions of the dynamic behavior of the compressible liquid around an externally forced single bubble. In Sec. II the governing equations, boundary, and initial conditions are presented; the numerical method used to integrate the system is also described. Section III discusses the numerical results of the present model and compares them with those obtained via RP-type equations. Conclusions are detailed in Sec. IV.

\section{PROPOSED MODEL}

For a compressible Newtonian pure liquid the governing equations for continuity, momentum and energy for spherically symmetric motions are

$$
\begin{aligned}
\frac{1}{\rho_{l} c_{l}^{2}} \frac{D p_{l}}{D t}- & \beta_{l} \frac{D T_{l}}{D t}=-\frac{1}{r^{2}} \frac{\partial r^{2} u_{l}}{\partial r}, \\
\rho_{l} \frac{D u_{l}}{D t}= & -\frac{\partial p_{l}}{\partial r}+\frac{4}{3 r^{2}} \frac{\partial}{\partial r}\left(r^{2} \mu_{l} \frac{\partial u_{l}}{\partial r}\right) \\
& -\frac{8}{3} \frac{\mu_{l} u_{l}}{r^{2}}-\frac{4}{3} \frac{u_{l}}{r} \frac{\partial \mu_{l}}{\partial r}, \\
\rho_{l} c_{p} \frac{D T_{l}}{D t}= & \beta_{l} T_{l} \frac{D p_{l}}{D t}+\frac{1}{r^{2}} \frac{\partial}{\partial r}\left(r^{2} k_{l} \frac{\partial T_{l}}{\partial r}\right) \\
& +\frac{4}{3} \mu_{l}\left(\frac{\partial u_{l}}{\partial r}-\frac{u_{l}}{r}\right)^{2},
\end{aligned}
$$

where the subscript $l$ denotes the liquid phase, $D \chi / D t$ represents the substantial derivative of any arbitrary variable $\chi$, $c_{l}$ is the speed of sound, $\rho$ is the density, $p_{l}$ is the pressure, $\beta_{l}$ is the thermal expansion coefficient, $T_{l}$ is the temperature, $u_{l}$ is the radial velocity, $\mu_{l}$ is the dynamic liquid viscosity coefficient, $c_{p}$ is the specific heat at constant pressure, $\kappa_{l}$ is the thermal conductivity of the liquid, and $r$ and $t$ stand for the radial coordinate and time, respectively.

The transport equations for a mixture of perfect nonreacting gases, obeying the Navier-Poisson and Fourier law constitutive relations, and neglecting radiative heat transfer, are

$$
\begin{aligned}
\frac{D \rho_{b}}{D t}+\frac{\rho_{b}}{r^{2}} \frac{\partial r^{2} u_{b}}{\partial r}=0, & \\
\rho_{b} \frac{D u_{b}}{D t}= & -\frac{\partial p_{b}}{\partial r}+\frac{4}{3 r^{2}} \frac{\partial}{\partial r}\left(r^{2} \mu_{b} \frac{\partial u_{b}}{\partial r}\right) \\
& -\frac{8}{3} \frac{\mu_{b} u_{b}}{r^{2}}-\frac{4}{3} \frac{u_{b}}{r} \frac{\partial \mu_{b}}{\partial r},
\end{aligned}
$$

$$
\begin{gathered}
\rho_{b} \bar{c}_{p, b} \frac{D T_{b}}{D t}=\frac{D p_{b}}{D t}+\frac{1}{r^{2}} \frac{\partial}{\partial r}\left(r^{2} k_{b} \frac{\partial T_{b}}{\partial r}\right) \\
+\frac{4}{3} \mu_{b}\left(\frac{\partial u_{b}}{\partial r}-\frac{u_{b}}{r}\right)^{2}, \\
\rho_{b}=\rho_{b} \frac{R^{0}}{\bar{W}} T_{b},
\end{gathered}
$$

where the subscript $b$ denotes bubble variables and properties, $R^{0}$ is the universal perfect gas constant, and $\bar{W}$ is the averaged molecular weight of the gas. Equation (7) is applied to both condensable and non-condensable gases, and, thus, the water vapor is considered as one of the components.

The following boundary conditions are used. At the bubble center $(r=0)$, under spherical symmetry, derivatives of the gas variables are set to zero,

$$
\frac{\partial \rho_{b}}{\partial r}=\frac{\partial T_{b}}{\partial r}=\frac{\partial p_{b}}{\partial r}=0
$$

The velocity at $r=0$ must also vanish

$$
u_{b}=0 .
$$

At infinity, $r=R_{\infty} \gg R_{0}$, the liquid temperature far away from the bubble is taken as a constant

$$
T_{l}=T_{\infty} \text { (constant) } .
$$

For the forcing acoustic wave, either oscillating pressure or velocity far away from the bubble can be specified. In this work a sinusoidal pressure is imposed

$$
p_{l}\left(r=R_{\infty}, t\right)=p_{l, \infty}+\Delta p_{\infty} \sin (2 \pi f t),
$$

where $\Delta p_{\infty}$ is the wave amplitude induced, for example, by the pressure transducer and $p_{l, \infty}$ is the hydrostatic pressure.

At the interface $[r=R(t)]$, mass transfer is neglected, limiting the strict model applicability to forcing pressure wave frequencies above $100 \mathrm{kHz}$, where RP models have shown that the bubble dynamics is unaffected by evaporation/condensation. ${ }^{26-29}$ Viscous stresses and radiation heat transfer are also neglected. Continuity, momentum, and energy transport equations applied to an infinitesimal shell about the interface yield the following relations, coupling bubble and liquid variables,

$$
\begin{aligned}
& \dot{R}=u_{l}=u_{b}, \\
& p_{b}(r=R)=p_{l}(r=R)+\frac{2 \sigma}{R(t)}, \\
& k_{b} \frac{\partial T_{b}}{\partial r}=k_{l} \frac{\partial T_{1}}{\partial r},
\end{aligned}
$$

where $\dot{R}$ is the interface velocity, $\sigma$ is the surface tension, and $\kappa_{b}$ and $k_{l}$ are the gas and liquid thermal conductivity, respectively. 
Continuity of temperature profiles at the interface is also assumed

$$
T_{b}=T_{l}
$$

At the initial time, $t=t_{0}$, the existence in the liquid of cavitation nuclei of a given radius is assumed. These gas nuclei are considered to be in equilibrium with the surrounding liquid. Thus, the initial bubble radius, $R_{0}$, is known, and the initial velocity is zero. The initial temperature of the bubble is equal to that of the liquid (thermal equilibrium),

$$
T_{l}\left(t=t_{0}\right)=T_{b}\left(t=t_{0}\right)
$$

The initial bubble pressure is obtained in terms of the initial liquid pressure from the continuity of the radial stress (mechanical equilibrium) at the interface, using the YoungLaplace equation

$$
p_{b}\left(r, t_{0}\right)=p_{l}\left(r=R, t_{0}\right)+\frac{2 \sigma}{R_{0}} .
$$

The initial density can be obtained from the equation of state for a mixture of perfect gases given the temperature, pressure, and composition.

\section{NUMERICAL METHOD}

To solve the system of Eqs. (4)-(17) the following set of unknown variables is defined:

$$
\boldsymbol{Y}=\left(\boldsymbol{p}_{g}, \boldsymbol{u}_{g}, \boldsymbol{T}_{g}, \boldsymbol{p}_{l}, \boldsymbol{u}_{l}, \boldsymbol{T}_{l}\right)
$$

The wide range of time scales present in the bubble expansion and implosion processes requires the use of adaptive stepsize control. In particular, in the numerical code a fifth order Cash-Karp Runge-Kutta method with local truncation error monitoring has been utilized to adjust the stepsize and ensure accuracy. ${ }^{30}$

The initial boundary conditions determine the initial boundary value problem. In particular, when variables $\left(\boldsymbol{Y}^{t}\right)$ are known at some arbitrary time $t$, the temporal integration supplies the solution $\left(\boldsymbol{Y}^{t+h}\right)$ at $t+h$, where $h$ is the time step.

The stepsize control is achieved using the solution of a fifth order Runge-Kutta method and that of a fourth order Runge-Kutta method with the same intermediate points as those used in the fifth order method. As a result, no extra evaluations are required. Estimating the error using both solutions, which theoretically scales as $h^{5}$, the stepsize is controlled.

The Runge-Kutta method is based on the evaluation of the derivatives in different intermediate points of the temporal stepsize. For each intermediate point, the resolution strategy is as follows:

(1) Properties are evaluated at the time in which the value of $\boldsymbol{Y}_{, t}$ is required. The calculation of the properties includes the gas density, which is computed from the obtained values for the gas pressure and temperature (note that instead of the density, the pressure is used as a primitive variable for the gas).
(2) The continuity, momentum, and energy equations in both phases [Eqs. (1)-(6)] are solved using an atomic layer epitaxy arbitrarian Eulerian Lagrangian (ALE) stabilized finite element method (FEM). ${ }^{31}$ Let the spatial domain be denoted by $\Omega$ and its boundary by $\Gamma$. The domain is subdivided into $n_{\mathrm{el}}$ elements $\Omega^{e}$. The variational formulation is defined for the set of variables $\boldsymbol{Y}$, according to Hauke and Hughes. ${ }^{32,33}$ Given a trial solution space $S_{Y}$, a weighting function space $\mathcal{V}$, and the solution at time $t$, this formulation yields the solution $\boldsymbol{Y} \in S_{Y}$ at time $t+h$ such that for all $\boldsymbol{W} \in \mathcal{V}$,

$$
\begin{aligned}
\int_{\Omega}( & \left.\boldsymbol{W} \cdot \boldsymbol{U}_{, t}(\boldsymbol{Y})-\boldsymbol{W},{ }_{i} \cdot \boldsymbol{F}_{i}^{\mathrm{adv}}(\boldsymbol{Y})+\boldsymbol{W}_{, i} \cdot \boldsymbol{K}_{i j} \boldsymbol{Y},{ }_{j}-\boldsymbol{W} \cdot \boldsymbol{S}\right) d \boldsymbol{\Omega} \\
& +\sum_{e=1}^{n_{\mathrm{el}}} \int_{\Omega^{e}}\left(\mathcal{L}^{T} \boldsymbol{W}\right) \cdot \tau(\mathcal{L} \boldsymbol{Y}-\boldsymbol{S}) d \Omega \\
= & \int_{\Gamma} \boldsymbol{W} \cdot\left(-\boldsymbol{F}_{i}^{\mathrm{adv}}(\boldsymbol{Y})+\boldsymbol{F}_{i}^{\mathrm{diff}}(\boldsymbol{Y})\right) n_{i} d \Gamma
\end{aligned}
$$

where the summation convention is used, $\boldsymbol{F}_{i}^{\mathrm{adv}}$ is the $i$ th convective flux, $\boldsymbol{F}_{i}^{\text {diff }}$ the $i$ th diffusive flux, $n_{i}$ is the $i$ th component of the normal vector, and $\boldsymbol{S}$ the source term. $\boldsymbol{K}_{i j}$ denotes the diffusion matrices. The first and last integrals constitute the Galerkin terms expressed as a function of the variables $\boldsymbol{Y}$, written in conservative form to ensure that the weak solution is bestowed with the correct Rankine-Hugoniot relations.

The least-squares contribution is written in terms of the differential operators $\mathcal{L}$ and $\mathcal{L}^{T}$, which, respectively, are given by

$$
\mathcal{L}=\boldsymbol{A}_{0} \frac{\partial}{\partial t}+\boldsymbol{A}_{i} \frac{\partial}{\partial x_{i}}-\frac{\partial}{\partial x_{i}}\left(\boldsymbol{K}_{i j} \frac{\partial}{\partial x_{j}}\right)
$$

and

$$
\mathcal{L}^{T}=\boldsymbol{A}_{0}^{T} \frac{\partial}{\partial t}+\boldsymbol{A}_{i}^{T} \frac{\partial}{\partial x_{i}}-\frac{\partial}{\partial x_{i}}\left(\boldsymbol{K}_{i j}^{T} \frac{\partial}{\partial x_{j}}\right),
$$

where $A_{i}^{\prime} s$ are the Euler Jacobians. The stabilizing matrix is denoted by $\tau$ and definitions can be found in Refs. 34 and 35 . Here, the diagonal version ${ }^{36-38}$ has been employed.

Because transient processes are taken into account, a temporal integration method is required. The finite element method can be expressed as

$$
\boldsymbol{M Y}{ }_{, t}+\boldsymbol{K Y}=\boldsymbol{F}
$$

For practical reasons, an implicit method is used

$$
\boldsymbol{M Y}{ }_{t}+\boldsymbol{K}^{t+\Delta t} \boldsymbol{Y}^{t+\Delta t}=\boldsymbol{F}^{t+\Delta t}
$$

This method provides a greater robustness than explicit ones, which translates into larger temporal steps and a substantial saving of central processing unit (CPU) time. The temporal derivative is approximated by

$$
\boldsymbol{Y}^{t+\Delta t}=\boldsymbol{Y}^{t}+\Delta t \boldsymbol{Y}_{, t}
$$

and Eq. (23) can then be rearranged as

$$
\boldsymbol{Y}_{, t}=[\boldsymbol{M}+\boldsymbol{K} \Delta t]^{-1}\left[\boldsymbol{F}-\boldsymbol{K} \boldsymbol{Y}^{t}\right] .
$$


The main disadvantage of this method is that the matrices $\boldsymbol{M}$ and $\boldsymbol{K}$ and the vector $\boldsymbol{F}$ should be evaluated at $t+\Delta t$. The values at $t+\Delta t$ are extrapolated with the values of the derivatives obtained in the previous step (for further details see Ref. 28).

The system of equations represented by Eq. (25) is decoupled as follows:

(a) The coupled liquid and gas energy equations, Eqs. (3) and (6), are first solved inside the bubble and the surrounding liquid.

(b) The continuity and momentum equations, Eqs. (1), (2), (4), and (5), inside the bubble and the surrounding liquid are also solved using an ALE stabilized FEM method. As the liquid is considered to be a low compressibility substance, it is not desirable to segregate the solution method. The strong coupling among the equations in both phases forces their solutions to be obtained simultaneously, which increases the CPU time.

It has been found that the best strategy consists of using the pressure as the primitive variable in both phases. When pressure is selected in the gas as a primitive variable and the continuity and momentum equations are solved, a non-linear term appears in the gas continuity equation

$$
\frac{1}{p_{g}} \frac{D p_{g}}{D t}-\beta_{T} \frac{D T_{g}}{D t}=-\frac{1}{r^{2}} \frac{\partial r^{2} u_{g}}{\partial r},
$$

where $\beta_{T}=1 / T_{g}$ for a perfect gas. In order to avoid this nonlinearity, the term $1 / p_{g}$ has been lagged, being evaluated at time $t$ instead of considering it as an unknown variable.

In this case, there are two unknown variables, the pressure and the velocity, per equation. In the equation of the node at the interface, liquid and gas velocities are equal [Eq. (12)]. However a pressure jump at the interface appears, and the liquid pressure there as a function of the gas pressure must be obtained from the local force balance [Eq. (13)], in which the viscous stresses have been neglected. The result is a heptagonal system of equations from which the velocity and pressure temporal derivatives in the liquid and in the gas can be worked out. Even though the solution of this type of system is more expensive than that of tridiagonal systems, the computational cost is still affordable using appropriate strategies for band diagonal systems included in Ref. 39, resulting in a much more robust technique.

(3) When the complete equations are solved inside and outside the bubble the interface velocity is supplied by the velocity field obtained in the previous step.

\section{NUMERICAL RESULTS}

\section{A. Standing waves in a pure liquid}

In the absence of a bubble, it is possible to derive an analytical solution for standing spherical pressure waves in a spherical flask. The inviscid Euler equation with negligible inertia terms and the appropriate mass conservation can be written as ${ }^{40}$

$$
\frac{1}{\rho_{l} c^{2}} \frac{\partial p_{l}}{\partial t}=-\frac{1}{r^{2}} \frac{\partial\left(u_{l} r^{2}\right)}{\partial r},
$$

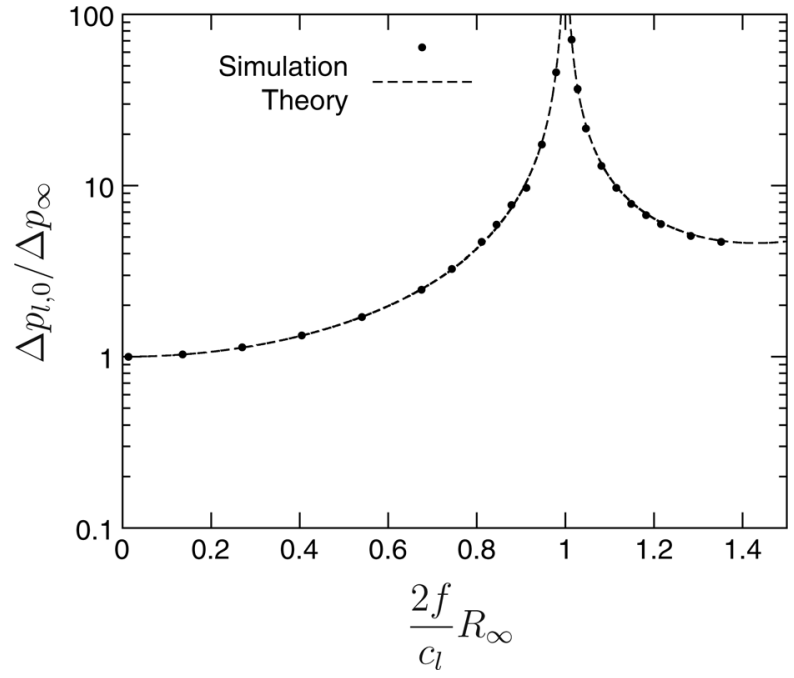

FIG 1. Comparison of the numerical and analytical solution of the amplitude of the pressure wave at $r=0$ as a function of $R_{\infty} . \Delta p_{\infty}=1 \mathrm{~atm}$ and $f=10000 \mathrm{~Hz}$.

$$
\rho_{l} \frac{\partial u_{l}}{\partial t}=-\frac{\partial p_{l}}{\partial r}
$$

The velocity is zero at the origin and the liquid pressure is imposed at a distance $R_{\infty}$

$$
\begin{aligned}
& r=0 \quad u_{l}(0, t)=0, \\
& r=R_{\infty} \quad p_{l}\left(R_{\infty}, t\right)=p_{l, \infty}+\Delta p_{\infty} \sin (\omega t) .
\end{aligned}
$$

The standing wave solution is ${ }^{40}$

$$
\begin{aligned}
u_{l}(r, t)= & \frac{\Delta p_{\infty}}{\rho_{l} c} \frac{1}{\sin \left(k R_{\infty}\right)} \frac{R_{\infty}}{r}\left(\cos (k r)-\frac{\sin (k r)}{k r}\right) \\
& \times \cos (\omega t), \\
p_{l}(r, t)= & p_{l, \infty}+\Delta_{p \infty} \frac{1}{\sin \left(k R_{\infty}\right)} \frac{R_{\infty}}{r} \sin (k r) \\
& \times \sin (\omega t),
\end{aligned}
$$

where $\omega=2 \pi f$ and the wavenumber $k=\omega / c_{l}$. At $r=0$ the amplitude of the standing pressure wave, $\Delta p_{l, 0}$, is

$$
\frac{\Delta p_{l, 0}}{\Delta p_{\infty}}=\frac{k R_{\infty}}{\sin \left(k R_{\infty}\right)} .
$$

Equation (32) implies that $\Delta p_{l, 0} / \Delta p_{\infty} \rightarrow 1$ for $\left(2 f / c_{l}\right) R_{\infty} \rightarrow 0$ and predicts resonance for $\left(2 f / c_{l}\right) R_{\infty}=1$.

The analytical and the numerical solutions for the dimensionless standing pressure wave amplitude at $r=0$ in water forced with a frequency of $10 \mathrm{kHz}$ as a function of $\left(2 f / c_{l}\right) R_{\infty}$ are depicted in Fig. 1 (the values of the parameters are contained in Table I). The oscillating pressure amplitude at the boundary has been set equal to $1 \mathrm{~atm}$. Both solutions are practically identical and this test serves as a validation of the code, at least, regarding the propagation of pressure waves in the liquid. 
TABLE I. Parameters of the numerical simulations.

\begin{tabular}{lccc}
\hline \hline$\mu_{l}$ & $\rho_{l}$ & $c_{l}$ & $f$ \\
\hline $8 \times 10^{-4} \mathrm{~kg} /(\mathrm{m} \mathrm{s})$ & $1000 \mathrm{~kg} / \mathrm{m}^{3}$ & $1480 \mathrm{~m} / \mathrm{s}$ & $10000 \mathrm{~Hz}$ \\
\hline \hline
\end{tabular}

The standing pressure wave amplitude at the flask center depends on $R_{\infty}$. For that reason, should a bubble at $r=0$ be present, conditions for the most intense implosions depend not only on the bubble resonance frequency but also on that of the vessel.

\section{B. Weak bubble oscillations}

When a single bubble in a compressible liquid is considered, no analytical solutions are available and one must resort to numerical integration of the conservation equations.

Solutions provided by RP models should, in principle, converge to those of the present model for low amplitudes of the forcing pressure wave, where liquid compressibility effects are expected to be negligible. It is thus interesting to verify the coincidence of the present results with those predicted by RP models, typically governed by the equation

$$
R \ddot{R}+\frac{3}{2} \dot{R}=\frac{1}{\rho l}\left(p_{l}(r=R, t)-p_{l, \infty}(t)\right),
$$

where $p_{l, \infty}(t)$ is the pressure at a radial distance far away from the bubble, liquid compressibility effects being negligible. Due to the low amplitudes tested in this section, all RP-type equations should tend to the same solution.

For a comparison among the different models, $p_{l, \infty}(t)$ in Eq. (33) and other RP-type equations can, in principle, be approximated by the solution of Eq. (31) evaluated at $r=0$. Except for very high frequencies, the bubble radius is much smaller than a wavelength and therefore, the pressure at $r=R_{\infty}$, required as a boundary condition in the new model, can be obtained from $\Delta p_{l, 0}$ using Eq. (32). In other words, given the amplitude of the pressure at the flask center, one can use Eq. (32) to impose the appropriate boundary condition to compare models.

In the current model, the boundary condition must be imposed as far as possible from the bubble in order to avoid any influence of $R_{\infty}$ on the solution. To estimate the minimum radius, $R_{\infty}$, for imposing the boundary condition with no effect on the bubble radius evolution, one can take $U_{c, 1}$ $=\Delta p_{\infty} /\left(\rho_{l} c_{l}\right)$ as a characteristic velocity induced in the liquid by a forcing pressure wave. Should the liquid in the presence of an oscillating bubble be assumed incompressible, its velocity at $R_{\infty}$ would be

$$
U_{c, 2}=\dot{R}\left(\frac{R_{b}}{R_{\infty}}\right)^{2}
$$

As the velocity due to the bubble oscillation becomes much smaller than the forcing pressure wave induced velocity, $U_{c, 2} \ll U_{c, 1}$, the influence of the bubble in the modification of the local velocity field is negligible. This condition implies that for

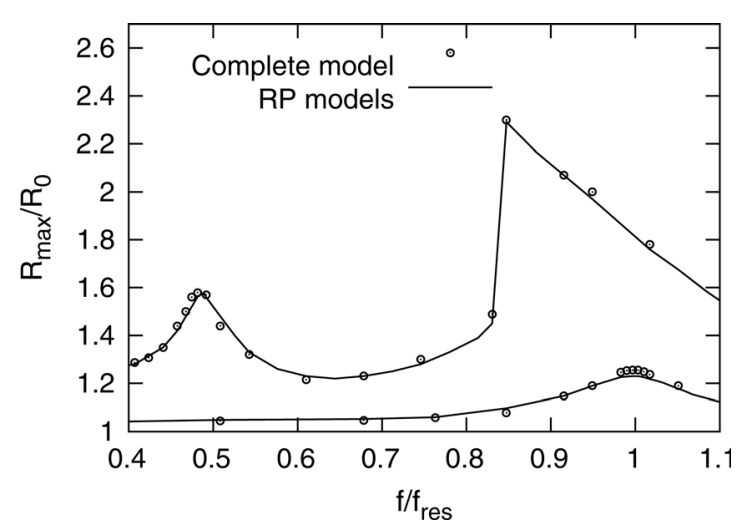

FIG. 2. Maximum bubble radius as a function of the excitation frequency for $\Delta p_{\infty}=0.1$ and $0.5 \mathrm{~atm}$. Results for the complete model and the model using the RP equation.

$$
R_{\infty} \gg \sqrt{\frac{\rho_{l} c_{l} \dot{R}}{\Delta p_{\infty}}} R_{0},
$$

one can expect a good agreement between RP models and the present one.

At low amplitudes of the forcing pressure wave this condition is easily satisfied when $R_{\infty}$ is chosen to be at a pressure antinode. In this case, $\Delta p_{\infty}$ is much larger than the pressure disturbance induced by the bubble at $R_{\infty}$ and it is possible to find out a priori the amplitude of the pressure wave applying Eq. (32). Thus, attention is first focused on small excitation cases. Figure 2 depicts the values of $R_{\max } / R_{0}$ predicted by both the standard RP model and the present one, for an air bubble with an initial radius of $10 \mu \mathrm{m}$ immersed in water forced ultrasonically with $\Delta p_{\infty}$ equal to 0.1 and $0.5 \mathrm{~atm}$, respectively. The natural frequency in the linear regime, ${ }^{41}$

$$
\omega_{p}=2 \pi f_{\mathrm{res}}=\left[\frac{3 k_{p} p_{\infty}}{\rho_{l} R_{0}^{2}}+\frac{2\left(3 k_{p}-1\right) \sigma}{\rho_{l} R_{0}^{3}}-\frac{8 v_{l}}{R_{0}^{4}}\right]^{1 / 2},
$$

has been used to make frequencies dimensionless in Fig. 2, where $v_{l}$ is the kinematic viscosity of the liquid. This expression depends on the polytropic coefficient, $k_{p}$, which, for air, may vary from 1 to 1.4. Thus, the natural frequency $\left(f_{\text {res }}\right)$ ranges between 290000 and $340000 \mathrm{~Hz}$. A numerical calculation of the amplitude of the bubble oscillation as a function of the forcing frequency for an amplitude of $\Delta p_{\infty}=0.1 \mathrm{~atm}$ reveals that the natural frequency is around $3 \times 10^{5} \mathrm{~Hz}$.

As expected, good agreement between the two models is found for the range of frequencies tested here. The errors of the RP model always remain below $5 \%$ for small amplitudes, which validates the code and also shows the convergence of the present model to the solution in the incompressible limit.

The new model also allows calculation of the pressure field in the liquid surrounding the bubble. As an example, the oscillation of a $10 \mu \mathrm{m}$ air bubble in water forced ultrasonically at the natural frequency of the bubble $\left(3 \times 10^{5} \mathrm{~Hz}\right)$. The amplitude of the pressure imposed at the boundary of the domain is set to generate a standing wave of $1.5 \mathrm{~atm}$ 

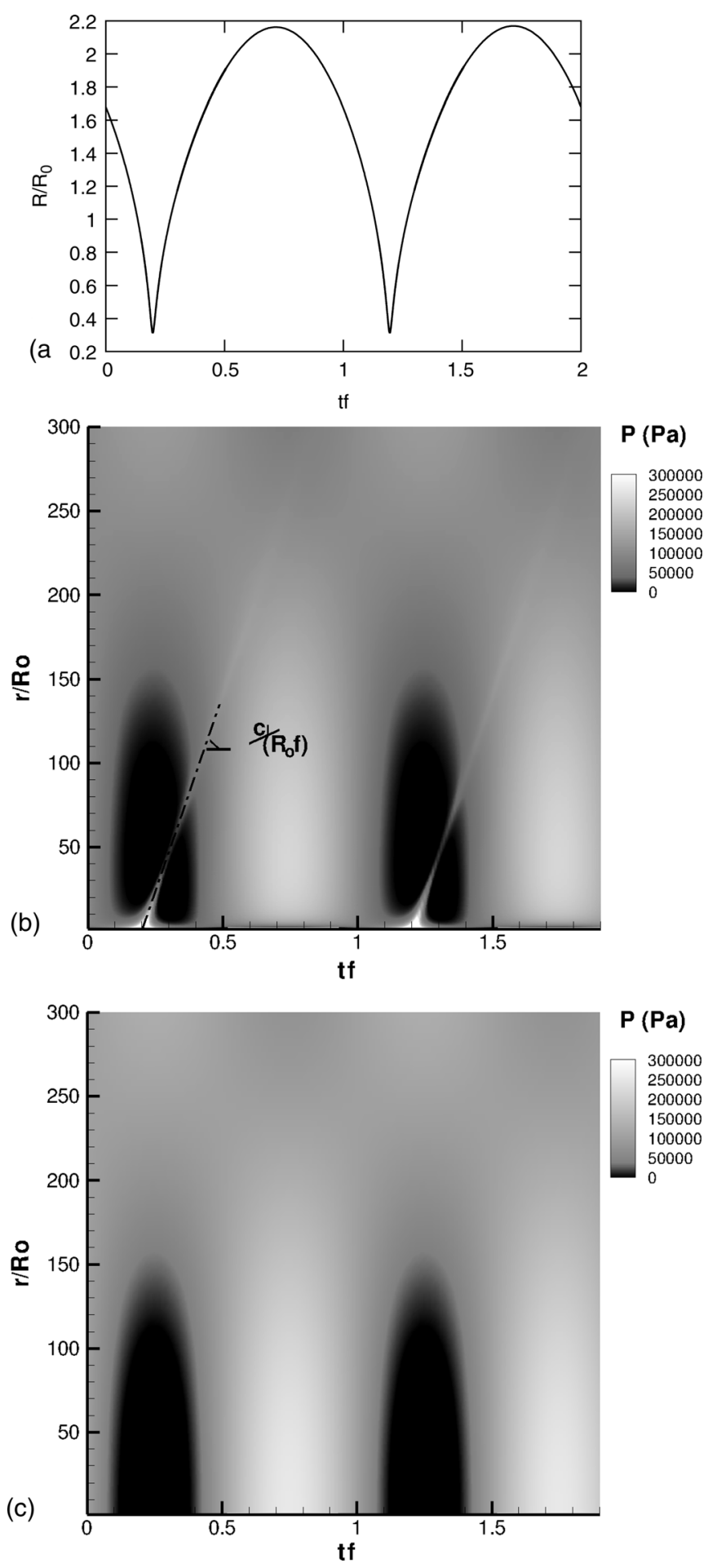

FIG. 3. Temporal evolution of the bubble radius evolution (up) and radial pressure distribution for a $10 \mu \mathrm{m}$ air bubble in water excited with a frequency of $3 \times 10^{5} \mathrm{~Hz}$ in an spherical flask with bubble (middle) and without bubble (bottom). The forcing pressure is set to generate a wave amplitude of $1.5 \mathrm{~atm}$ at the flask center when there is no bubble. The current model allows capturing pressure waves emitted during the implosion as well as the pressure disturbances induced by the bubble in the surrounding liquid.

at the flask center when there is no bubble. The value of the perturbation induced at the flask wall is obtained from Eq. (32). The radius of the flask is $R_{\infty}=0.01 \mathrm{~m}$. Figure 3 depicts the temporal evolution of the bubble radius and of the radial pressure distribution in the liquid with and without a bubble for two cycles. The solution without a bubble is directly computed using Eq. (31). The pressure fields are obtained after 200 cycles, when standing waves are clearly established in the flask and the bubble response is periodic. The two pressure fields contained in Fig. 3 show how the numerical solution obtained with bubble recovers the standing waves patterns expected from the analytical solution given by Eq. (31). Due to the spherical symmetry of the problem, the amplitude of the waves decays with the distance $r$. The presence of the bubble significantly modifies the pressure distributions in the liquid in a distance of the order of the bubble radius. During the bubble collapse, the pressure in the liquid is significantly influenced by the presence of the bubble up to distances of the order of 50 times the bubble radius. The collapse induces a pressure wave in the liquid propagating outward from the bubble and quickly decaying due to geometrical effects.

As the amplitude of the forcing pressure wave becomes larger, obtaining a periodic solution for the bubble response becomes more difficult. This is due to the appearance of long-lived transients in the numerical solutions, compounded by the interference of the driving pressure with reflections at the edge of the computational domain of pressure waves radiated by the bubble itself. This fact prevents a rigorous comparison of the model results with typical experimental setups like those of Wang et al. ${ }^{42}$

\section{Validity of approaches based on the RP equation during strong collapses}

The analysis of strong implosions, where the validity of the RP equation is questionable, is considered in this section. Pressures in the liquid region near to the bubble can reach values of the order of thousands of atmospheres for intense bubble collapses and then compressibility effects in the liquid become important. ${ }^{17,22,42}$ In those situations, peak pressures can be significantly influenced by other physical mechanisms like, for example, mass transfer, chemical reactions, and ionization. A rigorous comparison with experimental data would demand the inclusion of all possible relevant effects in the model. In this section, the accuracy of the RP-type equations is quantified under the assumption that some of these effects are negligible. This fact restricts the applicability of the current model and impedes a direct comparison with experiments. However, it still allows a quantitative estimation of compressibility effects as a function of the peak pressure at collapse.

In particular, the following equations are tested. $\mathrm{KM},{ }^{4}$

The RP equation with the modification proposed by

$$
\begin{aligned}
\left(1-\frac{\dot{R}}{c_{l}}\right) R \ddot{R}+\frac{3}{2}\left(1-\frac{\dot{R}}{3 c_{l}}\right) \dot{R}^{2} \\
=\frac{1}{\rho_{l}}\left(1+\frac{\dot{R}}{c_{l}}\right)\left(p_{l}(r=R)-p_{l, \infty}(t)\right) \\
\quad+\left.\frac{R(t)}{\rho_{l} c_{l}} \frac{\partial p_{l}(r, t)}{\partial t}\right|_{r=R(t)},
\end{aligned}
$$


the Gilmore equation

$$
\begin{aligned}
& \left(1-\frac{\dot{R}}{c_{l}}\right) R \ddot{R}+\frac{3}{2}\left(1-\frac{\dot{R}}{3 c_{l}}\right) \dot{R}^{2} \\
& =\frac{1}{\rho_{l}}\left(1+\frac{\dot{R}}{c_{l}}\right) H+\left.\left(1-\frac{\dot{R}}{c_{l}}\right) \frac{R(t)}{\rho_{l} c_{l}} \frac{\partial H}{\partial t}\right|_{r=R(t)},
\end{aligned}
$$

where

$$
H=\int_{p_{l, \infty}}^{p_{l}(r=R)} \frac{d p}{\rho},
$$

and the expression derived by Tomita and Shima, ${ }^{43}$ proven by Lezzi and Prosperetti ${ }^{19}$ to be second order accurate,

$$
\begin{aligned}
(1 & \left.-2 \frac{\dot{R}}{c_{l}}+\frac{23}{10}\left(\frac{\dot{R}}{c_{l}}\right)^{2}\right) R \ddot{R}+\frac{3}{2}\left(1-\frac{4}{3} \frac{\dot{R}}{c_{l}}+\frac{7}{5}\left(\frac{\dot{R}}{c_{l}}\right)^{2}\right) \dot{R}^{2} \\
= & \frac{p_{l}(r=R)-p_{l, \infty}(t)}{\rho_{l}}\left(1-\frac{3}{2} \frac{p_{l}(r=R)-p_{l, \infty}(t)}{\rho_{l} c_{l}^{2}}\right. \\
& \left.+\frac{1}{2}\left(\frac{\dot{R}}{c_{l}}\right)^{2}\right)+\left.\left(1-2 \frac{\dot{R}}{c_{l}}\right) \frac{R(t)}{\rho_{l} c_{l}} \frac{\partial p_{l}(r, t)}{\partial t}\right|_{r=R(t)}
\end{aligned}
$$

In order to compare the different models under conditions where compressibility effects become relevant, the following test is considered: A bubble with a given initial pressure, $p_{b, 0}$, at the reference temperature and zero velocity is initially assumed to be present in a liquid whose pressure at infinity is maintained constant and equal to

$$
p_{l, \infty}=p_{l, 0}+\Delta p_{\infty} .
$$

After a certain time, the bubble compresses to a new equilibrium pressure. This case can be an approximation of the conditions when the bubble attains its maximum radius in SBSL; it can also be representative of the interaction between a strong pressure wave and a gas bubble. In practical applications, when violent collapses are produced $\Delta p_{\infty} \gg p_{b, 0}$ and, therefore, one can take $p_{l, \infty} \sim \Delta p_{\infty}$.

The initial pressure difference between the bubble and the liquid at infinity produces an acceleration of the interface which, assuming an incompressible liquid, can be obtained from Eq. (33)

$$
\begin{aligned}
\ddot{R}(t=0) & =\frac{1}{R_{0} \rho_{l}}\left(p_{l, 0}\left(r=R_{0}\right)-p_{l, \infty}\right) \\
& =-\frac{1}{R_{0} \rho_{l}} \Delta p_{\infty} .
\end{aligned}
$$
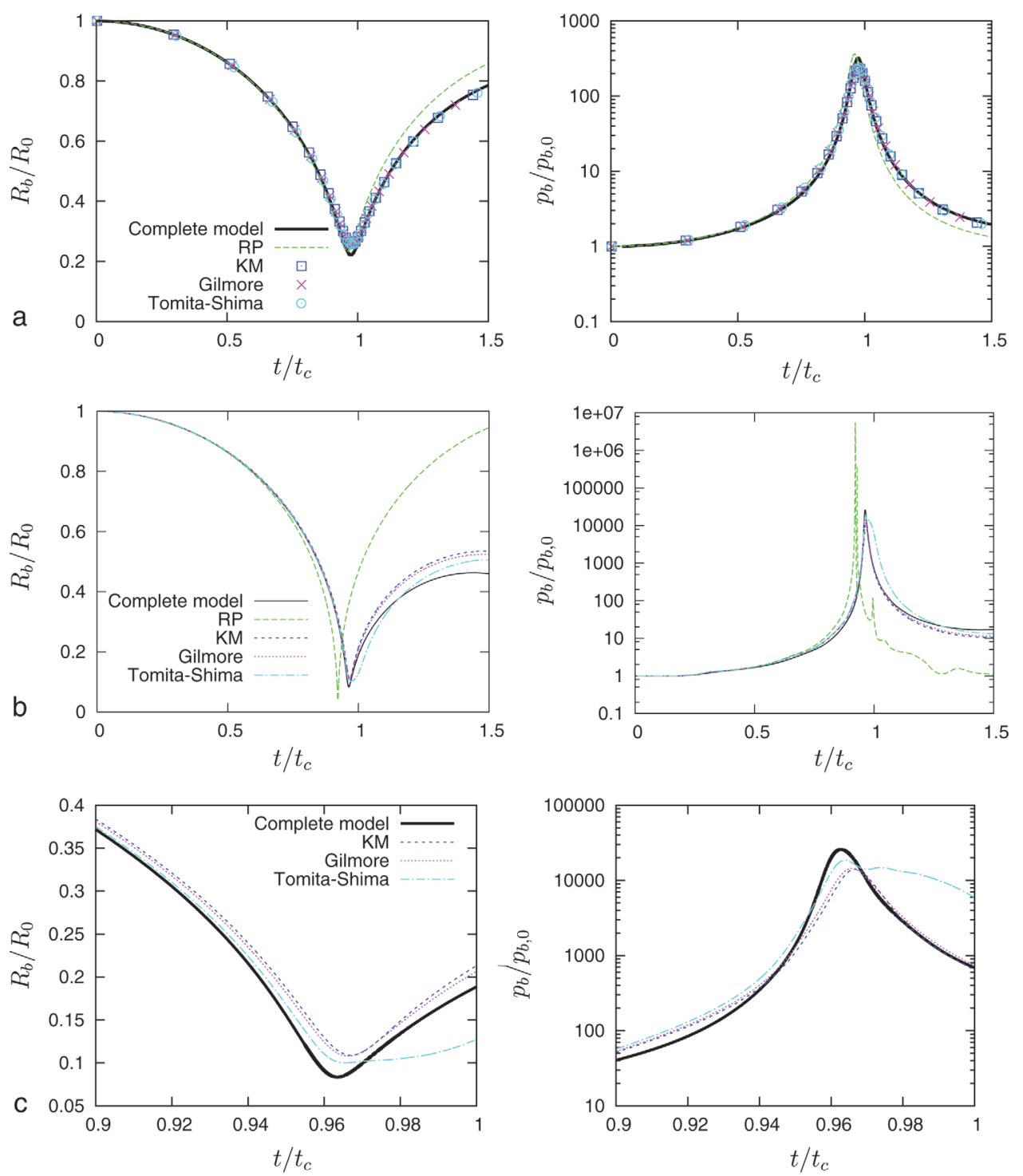

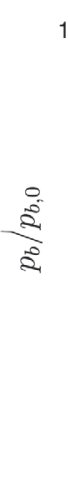

FIG. 4. (Color online) Bubble radius and bubble pressure (at $r=0$ ) evolutions as functions of dimensionless time for the complete model, the standard RP equation and the RP-type equations with the compressibility corrections suggested by KM, Gilmore and Tomita and Shima. The ratios between the liquid pressure at infinity and the initial bubble pressure, $p_{b, 0}$ are 10 (top) and 100 (center). At the bottom, a zoom of the implosion for a ratio equal to 100 is shown. The model proposed by Tomita and Shima displays the more accurate behavior before the collapse. 
Integrating the momentum equation in the liquid from the bubble interface to some arbitrary distance $r$, the following expression for the initial pressure field is readily obtained:

$$
p_{l}(r, t=0)=p_{b, 0}+\frac{2 \sigma}{R_{0}}-\rho_{l}\left(\ddot{R} R_{0}\left(1-\frac{R_{0}}{r}\right)\right) .
$$

The Rayleigh collapse time ${ }^{2}$ defined by ${ }^{41}$

$$
t_{c}=0.915 \sqrt{\frac{\rho_{l}}{p_{l, \infty}}} R_{0},
$$

can be taken as a convenient characteristic implosion time of a bubble in an incompressible liquid.

To test the validity of the models, an air bubble of $10 \mu \mathrm{m}$ at $p_{b, 0}=10^{5} \mathrm{~Pa}$ is introduced in water. The value of $p_{l, \infty}$, is used to control the bubble implosion intensity $\left(\Delta p_{\infty}\right.$ $\left.=p_{l, \infty}-p_{b, 0}\right)$.

The bubble radius and pressure (at $r=0$ ) evolutions as functions of the dimensionless time $t / t_{c}$ are shown in Fig. 4 for different bubble implosion intensities. Figure 5 depicts the peak pressures at the bubble center reached during the bubble collapse for each of the models tested in this work. The larger $p_{l, \infty}$, the stronger the implosion. As can be seen, the Rayleigh time correctly scales the implosion occurrence for the different cases. From Figs. 4 and 5, one might conclude that the RP-type models capture relatively well the bubble radius and pressure evolutions for implosions with peak pressures below 1000 atm. For more intense collapses, the corrected RP-type equations only predict the order of magnitude of the pressures reached during the collapse. As expected, the correction suggested by Tomita and Shima turns out to be the most accurate approximation, ${ }^{19}$ although the relative error for the most intense collapse tested here is still of the order of $100 \%$. Only right after the bubble

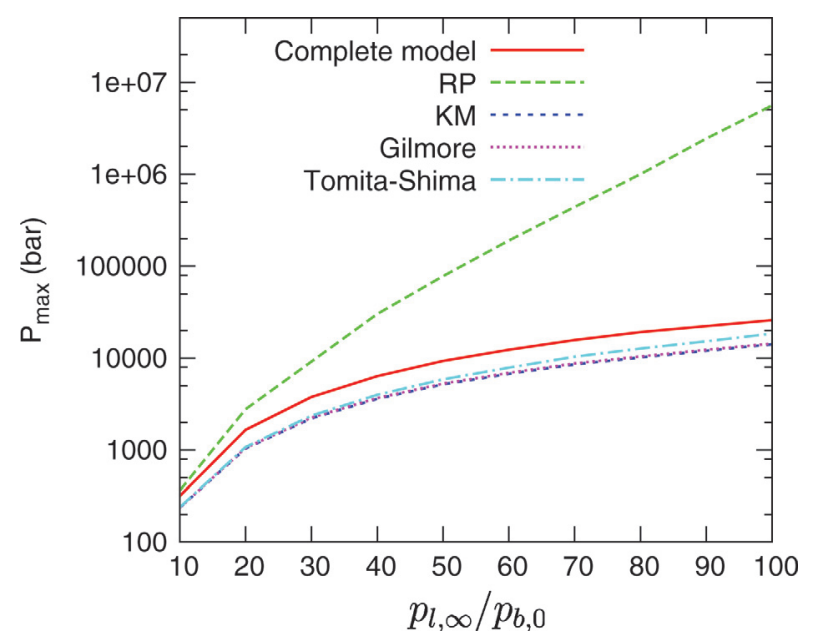

FIG. 5. (Color online) Peak pressures at the bubble center at the collapse obtained with the five models compared in this work for different ratios of the initial bubble pressure to the pressure at infinity. Models based on the RP equation accurately predict the peak pressures for values of the peak pressures below $1000 \mathrm{~atm}$. For extremely violent implosions, RP models dramatically fail predicting the peak pressures. The modified RP-type models studied in this work significantly improve predictions, especially that proposed by Tomita and Shima, but errors are still of the order of $100 \%$. collapse and during a very short time, the corrections introduced by Tomita and Shima do not properly capture the bubble radius evolution. The reason of this anomalous behavior can be attributed to the fact that the accuracy of the approximations introduced by the corrections are only proved to work for low Mach numbers. However, during the quick expansion produced after the intense collapse, the Mach number becomes of the order of 1 and the analysis presented by Lezzi et al. does not apply. For that reason, although the equation proposed by Tomita and Shima provides, overall, the most accurate bubble radius evolution, appreciable errors are encountered in the $R-t$ curves during the first instants of the expansion produced after the collapse. Compared to the corrected formulas, the present model predicts larger pressures during the collapse and lower amplitude of the rebound. That is, the corrections introduced in the RP equation seem to overestimate the energy dissipation before the collapse, whereas they tend to underestimate it after the bubble reaches its minimum radius. For intense collapses, the large pressures reached at the interface compress the liquid, absorbing in that process a significant fraction of the energy concentrated during the implosion. When the bubble starts to expand, part of that energy is invested in generating pressure waves in the liquid which are emitted from the bubble interface outward at every rebound. This wave is depicted in Fig. 6 for the case of $\Delta p_{l, \infty} / p_{b, 0}=100$. In general, it can be concluded that the energy absorbed by the outgoing wave is not correctly captured by the first and second order models tested here. Pressure values of the order of those attained inside the bubbles are reached at distances of the order of the bubble radius; the effect of these pressure waves is still apparent up to characteristic distances of the order of several times $R_{b}$.

Thus, liquid compressibility effects are crucial in order to get accurate estimations of the internal pressures reached inside the bubble, as well as the amplitude of the rebounds produced thereafter. During intense bubble collapses, the new model yields pressure values up to two orders of magnitude lower than those predicted under the incompressible hypothesis (standard RP equation). The modifications proposed

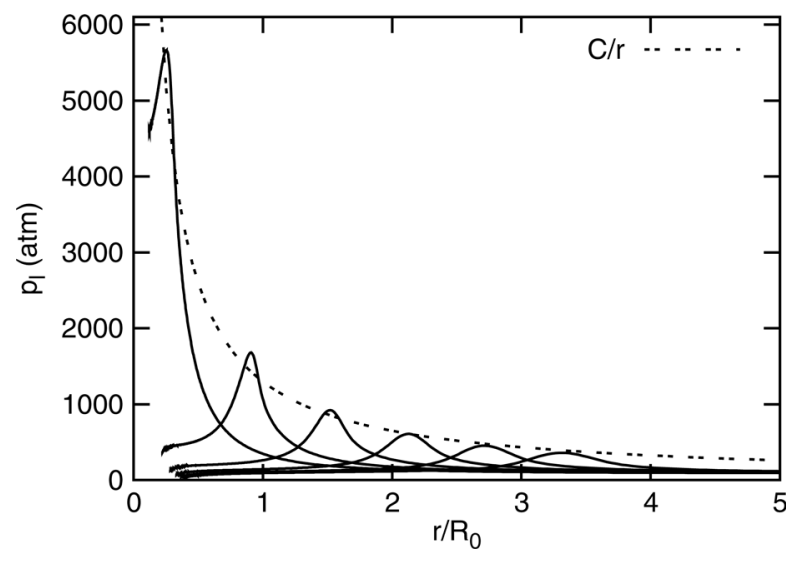

FIG. 6. Liquid pressure profiles after the bubble collapse every 4 ns. For large pressures, the wave dissipates energy and the amplitude decay is larger than $1 / r$. As the wave propagates outward from the bubble, its amplitude decreases, dissipation becomes less important and the shock wave amplitude is inversely proportional to the distance. 
by KM, Gilmore, and Tomita and Shima, to account for liquid compressibility effects, significantly improve the standard RP predictions; however, errors are still large for extremely violent implosions. Thus, the complete solution of the liquid continuity and momentum equations in the liquid is compulsory if accurate values are required. As a first approximation however, the modified versions of the RP equation can be used to obtain an approximate value of pressures reached during the collapse.

\section{CONCLUSIONS}

A spherically symmetric one-dimensional model of single bubble dynamics has been presented. The complete model solves the mass, momentum, and energy equations both inside the bubble and in the surrounding liquid. The model directly incorporates liquid compressibility effects, allowing the study of physical phenomena where small variations of the liquid density may play an important role. The new model has been numerically validated by comparison with the analytical solution of an ultrasonic field generating standing pressure waves within a spherical flask containing a compressible liquid without a bubble.

Predictions of the bubble radius amplitude as a function of frequency, obtained with both the standard RP model and the present one, have also been compared for low amplitude oscillations; a good agreement is apparent below, above, and at the bubble natural frequency, showing that liquid compressibility effects can be neglected in order to accurately predict the bubble dynamics.

For very intense collapses, however, where pressures at the bubble interface reach values above $1000 \mathrm{~atm}$, significant differences are observed between the predictions of the complete model and those based on the incompressible RP equation. Predicted peak pressure values are up to two orders of magnitude smaller than those calculated with the RP equation. The corrections suggested by KM, Gilmore, or Tomita and Shima significantly reduce the error to values around $100 \%$. As suggested by Lezzi and Prosperetti, ${ }^{19}$ the most accurate predictions are provided by the correction proposed by Tomita and Shima. ${ }^{43}$

The rigorous solution of the continuity and momentum equations in the liquid yields peak pressures larger than those obtained by RP-type models corrected to account for compressibility; although pressures are higher, the expansion radius reached during the first rebound is clearly smaller. Large pressures attained inside the bubble during the collapse generate pressure waves in the liquid which propagate outward. The effect of these propagation waves does not seem to be correctly captured by the first and second order corrections.

The inclusion of models accounting for effects missing in the current one, like mass transfer or chemical reactions, in future work should provide a more realistic correlation between pressures measured far from the bubble and those reached inside the bubble in SBSL experiments.

\section{ACKNOWLEDGMENTS}

The authors would like to acknowledge the comments and suggestions of Jean-Louis Thomas and Tim Colonius on a previous version of this manuscript. This work is a part of the Ph.D. dissertation of D. Fuster ${ }^{28}$ and has been partially supported by Ministerio de Ciencia y Tecnologia of Spain, under Grant No. CTM2004-061 84-C02-02.

${ }^{1} \mathrm{~W}$. Besant, A Treatise on Hydrostatics and Hydrodynamics (Deighton, Bell, London, 1859), p. 228.

${ }^{2} \mathrm{~L}$. Rayleigh, "On the pressure developed in a liquid during the collapse of a spherical cavity," Philos. Mag. 34, 94 (1917).

${ }^{3}$ F. R. Gilmore, "The growth of collapse of a spherical bubble in viscous compressible liquid," Technical Report No. 26-4, California Institute of Technology (1952).

${ }^{4}$ J. Keller and M. Miksis, "Bubble oscillations of large amplitude," J. Acoust. Soc. Am. 68, 628-633 (1980).

${ }^{5} \mathrm{M}$. Plesset and A. Prosperetti, "Bubble dynamics and cavitation," Annu. Rev. Fluid Mech. 9, 145 (1977).

${ }^{6}$ A. Prosperetti, "Bubble phenomena in sound fields: Part one," Ultrasonics 22, 69-77 (1984).

${ }^{7}$ A. Prosperetti, “A new mechanism for sonoluminescence," J. Acoust. Soc. Am. 101, 2003-2007 (1997).

${ }^{8} \mathrm{R}$. Toegel and D. Lohse, "Phase diagrams for sonoluminescing bubbles: A comparison between experiment and theory," J. Chem. Phys. 118, 1863-1875 (2003).

${ }^{9}$ Y. An and C. Ying, "Model of single bubble sonoluminescence," Phys. Rev. E 71, 1-12 (2005).

${ }^{10}$ K. Yasui, T. Tuziuti, M. Sivakumar, and Y. Iida, "Theoretical study of single-bubble sonochemistry," J. Chem. Phys. 122, 1-12 (2005).

${ }^{11}$ G. Hauke, D. Fuster, and C. Dopazo, "Dynamics of a single cavitating and reacting bubble," Phys. Rev. E 75, 1-14 (2007).

${ }^{12} \mathrm{~W}$. Moss, "Understanding the periodic driving pressure in the RayleighPlesset equation," J. Acoust. Soc. Am. 101, 1187-1190 (1997).

${ }^{13}$ S. Hilgenfeldt, M. Brenner, S. Grossmann, and D. Lohse, "Analysis of the Rayleigh-Plesset dynamics for sonoluminescing bubbles," J. Fluid Mech. 365, 171-204 (1998).

${ }^{14}$ H. Lin, B. D. Storey, and A. J. Szeri, "Inertially driven in homogeneities in violently collapsing bubbles: The validity of the Rayleigh-Plesset equation," J. Fluid Mech. 452, 145-162 (2002).

${ }^{15}$ A. Preston, T. Colonius, and C. Brennen, "A reduced order model of diffusive effects on the dynamics of bubbles," Phys. Fluids 19, 1-19 (2007).

${ }^{16}$ R. Nigmatulin, I. Akhatov, N. Vakhitova, and R. Lahey, "On the forced oscillations of a small gas bubble in a spherical liquid-filled flask," J. Fluid Mech. 414, 47-73 (2000).

${ }^{17}$ E. Johnson and T. Colonius, "Compressible multicomponent flow calculations and shock-wave interactions," in Sixth International Symposium on Cavitation (2006).

${ }^{18}$ A. Prosperetti and A. Lezzi, "Bubble dynamics in a compressible liquid. Part 1. First-order theory," J. Fluid Mech. 168, 457-478 (1986).

${ }^{19}$ A. Lezzi and A. Prosperetti, "Bubble dynamics in a compressible liquid. Part 2. Second-order theory," J. Fluid Mech. 185, 289-321 (1987).

${ }^{20} \mathrm{E}$. Johnsen and T. Colonius, "Implementation of WENO schemes in compressible multi-component flow problems," J. Comput. Phys. 219, 715-732 (2006).

${ }^{21}$ Z. Wang, R. Pecha, B. Gompf, and W. Eisenmenger, "Single bubble sonoluminescence: Investigations of the emitted pressure wave with a fiber optic probe hydrophone," Phys. Rev. E 59, 1777 (1999).

${ }^{22} \mathrm{R}$. Pecha and B. Gompf, "Microimplosions: Cavitation collapse and shock wave emission on a nanosecond time scale," Phys. Rev. Lett. 84, 1328-1330 (2000).

${ }^{23}$ W. Lauterborn, T. Kurz, R. Geisler, D. Schanz, and O. Lindau, “Acoustic cavitation, bubble dynamics and sonoluminiescence," Ultrason. Sonochem. 14, 484-491 (2007).

${ }^{24}$ G. Reisman, Y. Wang, and C. Brennen, "Observations of shock waves in cloud cavitation,” J. Fluid. Mech. 344, 255 (1998).

${ }^{25}$ Y. Wang, "Shock waves in bubbly cavitating flows," Ph.D. thesis, California Institute of Technology, Pasadena, CA, 1996.

${ }^{26} \mathrm{~A}$. Colussi, L. Weavers, and M. Hoffmann, "Chemical bubble dynamics and quantitative sonochemistry,” J. Phys. Chem. A 102, 6927-6934 (1998).

${ }^{27}$ G. Puente and F. Bonetto, "Proposed method to estimate the liquid-vapor accommodation coefficient based on experimental sonoluminescence data," Phys. Rev. E 71 (2005).

${ }^{28} \mathrm{D}$. Fuster, "Modeling and numerical simulation of the dynamics of liquidbubble cavitating systems with chemical reaction processes," Ph.D. thesis, Centro Politecnico Superior, Zaragoza, Spain, 2007. 
${ }^{29}$ D. Fuster, G. Hauke, and C. Dopazo, "Influence of accommodation coefficient on nonlinear bubble oscillations," J. Acoust. Soc. Am. 128, 5-10 (2010).

${ }^{30}$ W. Press, S. Teulkolsky, W. Vetterling, and B. Flannery, Numerical Recipes in Fortran 77 (Press Syndicate of the University of Cambridge, The Pitt Building, Trumpington Street, Cambridge CB2 1RP, 1992), Chap. 16.

${ }^{31}$ T. Hughes, W. Liu, and T. Zimmerman, "Langrangian-eulerian finite element formulation for incompressible viscous flows," Comput. Methods Appl. Mech. Eng. 29, 329-349 (1981).

${ }^{32} \mathrm{G}$. Hauke and T. Hughes, "A unified approach to compressible and incompressible flows," Comput. Methods Appl. Mech. Eng. 113, 389-395 (1994).

${ }^{33} \mathrm{G}$. Hauke and T. Hughes, "A comparative study of different sets of variables for solving compressible and incompressible flows," Comput. Methods Appl. Mech. Eng. 153, 1-44 (1998).

${ }^{34} \mathrm{~T}$. Hughes and M. Mallet, "A new finite element formulation for computational fluid dynamics: III. the generalized streamline operator for multidimensional advection-diffusion systems," Comput. Methods Appl. Mech. Eng. 58, 305-328 (1986).

${ }^{35} \mathrm{~F}$. Shakib, T. Hughes, and Z. Johan, "A new finite element formulation for fluid dynamics formulation: $X$. the compressible Euler and NavierStokes equations," Comput. Methods Appl. Mech. Eng. 89, 141-219 (1991).
${ }^{36}$ G. Hauke, "Simple stabilizing matrices for the computation of compressible flows in primitive variables," Comput. Methods Appl. Mech. Eng. 190, 6881-6893 (2001).

${ }^{37}$ G. Hauke, A. Landaberea, I. Garmendia, and J. Canales, "A segregated method for compressible flow computation part I: Isothermal compressible flows," Int. J. Numer. Methods Fluids 190, 271-323 (2004).

${ }^{38}$ G. Hauke, A. Landaberea, I. Garmendia, and J. Canales, "A segregated method for compressible flow computation. Part II: General divariant compressible flows," Int. J. Numer. Methods Fluids 49, 183-209 (2005).

${ }^{39}$ W. Press, S. Teulkolsky, W. Vetterling, and B. Flannery, Numerical Recipes in For tran 77 (Press Syndicate of the University of Cambridge, The Pitt Building, Trumpington Street, Cambridge CB2 1RP, 1992), Chap. 2.

${ }^{40} \mathrm{P}$. Morse and K. Ingard, Theoretical Acoustics, 927 (Princenton University Press, Princeton, NJ, 1986).

${ }^{41} \mathrm{C}$. Brennen, Cavitation and Bubble Dynamics (Oxford University Press, New York, 1995), p. 254.

${ }^{42}$ Z. Wang, R. Pecha, B. Gompf, and W. Eisenmenger, "Single bubble sonoluminescence: Investigations of the emitted pressure wave with a fiber optic probe hydrophone," Phys. Rev. E 59, 1777-1780 (1999).

${ }^{43}$ Y. Tomita and A. Shima, "On the behavior of a spherical bubble and the impulse pressure in a viscous compressible liquid," Bull. JSME 20, 14531460 (1977) 